\title{
A typology of personal factor attributes among shoppers
}

\begin{abstract}
Purpose: The purpose of this paper is to derive a comprehensive model with integrated dimensions of trait constructs to understand the shoppers' dispositional traits in consumption. This study endeavors to gain empirical validation of a motivational network of shoppers' traits in consumption as well as to ascertain different shoppers' typology from the configurations of personal factor attributes. Design/methodology/approach: Store-intercept method was used to collect data from a sample of 600 apparel adult shoppers at five shopping malls in Klang Valley, Malaysia. The factor structure of personal factors was achieved using confirmatory factory analysis. The hierarchical and non-hierarchical cluster analysis was employed to develop the shoppers' typology. Findings: A relatively good fit in confirmatory factor analysis validates the applicability of the conceptualized personal factor attributes measurement model. The constitution of personal factor attributes results in three shoppers typology of Confident, Enthusiastic Shoppers; Moderate, Pragmatic Shoppers and SelfConfined, Apathetic Shoppers. Practical implications: The study provides an understanding of the personal attribute factors and disseminates insightful information about profile of shoppers' typology. Accordingly, the implementation of the strategy which involving the personality and psychological desires of the consumers, is now possible. Originality/value: This paper stipulates new insights to discern other dimensions in personality traits to examine the personal factor attributes, by considering the elemental traits, compound traits, situational traits and surface traits in a holistic manner. The findings of this study advance the knowledge on personal factor attributes that shape shopping behavior along with practical applications.
\end{abstract}

Keyword: Apparel; Personality; Repatronage; Segmentation; Shopping; Typology 